\title{
SÉMANTIQUE DES ODEURS
}

Georges Kleiber, Marcel Vuillaume

\author{
Armand Colin | « Langages »
}

2011/1 n $181 \mid$ pages 17 à 36

ISSN 0458-726X

ISBN 9782200926809

Article disponible en ligne à l'adresse :

http://www.cairn.info/revue-langages-2011-1-page-17.htm

\section{Pour citer cet article :}

Georges Kleiber, Marcel Vuillaume« Sémantique des odeurs », Langages 2011/1 (n 181), p. 17-36.

DOI 10.3917/lang.181.0017

Distribution électronique Cairn.info pour Armand Colin.

(c) Armand Colin. Tous droits réservés pour tous pays.

La reproduction ou représentation de cet article, notamment par photocopie, n'est autorisée que dans les limites des conditions générales d'utilisation du site ou, le cas échéant, des conditions générales de la licence souscrite par votre établissement. Toute autre reproduction ou représentation, en tout ou partie, sous quelque forme et de quelque manière que ce soit, est interdite sauf accord préalable et écrit de l'éditeur, en dehors des cas prévus par la législation en vigueur en France. Il est précisé que son stockage dans une base de données est également interdit. 


\section{Georges Kleiber}

Université de Strasbourg \& LiLPa/Scolia (EA 1339)

\section{Marcel Vuillaume}

Université de Nice Sophia Antipolis \& Groupe MOD (Molécules, Olfaction, Discours)

\section{Sémantique des odeurs}

\section{EN GUISE D'INTRODUCTION}

Trois constats :

- sur le plan linguistique, les " odeurs » ont été beaucoup moins étudiées que les couleurs ${ }^{1}$. En jouant sur le titre - Un sens méconnu - du premier chapitre de l'ouvrage de J. Candau (2000), on pourrait parler du « sens méconnu d'un sens méconnu »;

- lorsqu'elles ont été abordées sous l'angle du langage, la problématique essentielle a été, comme pour les couleurs, celle de leur dénomination et classification/catégorisation ;

- une raison à cela, signalée dans quasiment tous les travaux linguistiques sur l'olfaction : les odeurs n'ont en général pas de dénominations spécifiques comme en ont les couleurs. Toutes les observations font état d'un langage des odeurs beaucoup plus pauvre, plus limité et plus instable, moins systématique que le langage d'autres domaines sensoriels comme le visuel ou l'acoustique ${ }^{2}$.

Une triple explication, d'ordre psycho-linguistique et neurophysiologique, a pu être avancée, mettant en jeu, comme nous l'avons mentionné dans notre présentation, la moindre importance du sens olfactif, les structures corticales du traitement des odeurs et l'absence, pour les odeurs, de classification selon des propriétés physiques ou chimiques.

Notre objectif n'est évidemment pas de nous placer sur un terrain qui n'est pas le nôtre. Ce sera l'aspect linguistique qui retiendra notre attention. Or, c'est surtout le domaine classificatoire, identificatoire qui constitue l'objet principal

1. Même si Candau (2000) écrit que « le langage des odeurs est en voie d'être sérieusement exploré ».

2. Voir Candau (2000 : 68) et Boisson (1997 : 31). 
des recherches (psycho-linguistiques), et les travaux menés, pour le français, par l'équipe de D. Dubois (voir les références bibliographiques) sont là pour en témoigner ${ }^{3}$. Mais, notre projet n'est pas d'examiner ici la pertinence de ces travaux et de nous placer dans leur prolongement. Ce qui constituera le principal sujet de notre enquête, c'est la sémantique même des termes, noms, verbes, etc. comme odeur, sentir, etc. Nous essaierons, en nous appuyant sur certaines de leurs propriétés, de déterminer le type d'entités que sont, pour les sujets parlants, les odeurs. Nous n'examinerons ici que la question des noms ${ }^{4}$ et ne traiterons qu'un seul point d'une problématique plus complexe et plus riche qu'il n'y parait. Nous limiterons, en effet, notre analyse sémantique des odeurs à la question du statut concret ou abstrait des odeurs, quitte à envisager d'autres questions dans des travaux séparés.

Mais avant de commencer, précisons l'idée qui sous-tend notre démarche. Nous pensons, à la suite de Z. Vendler (1967), que le langage est, pour reprendre sa formule, une sorte $\mathrm{d}^{\prime}$ « outil philosophique » qui non seulement donne accès aux catégories et distinctions conceptuelles au moyen desquelles nous pensons dans une langue donnée, mais les révèle. Autrement dit, appliquée aux odeurs ${ }^{5}$, cette idée signifie que l'analyse des propriétés et des contraintes qui caractérisent des noms comme odeur, parfum, arôme, etc. permet d'accéder au concept même d'odeur et, ainsi, de savoir ce que sont pour nous, ontologiquement, les odeurs. Il ne s'agit pas, comme on le croit parfois à tort, d'aboutir à des entités purement linguistiques : ce sont bien des entités non linguistiques qui sont visées et surtout déterminées par ces termes. Déterminer une propriété ou un trait à travers ses manifestations linguistiques n'implique nullement que la propriété ou le trait mis ainsi en relief soit aussi de nature linguistique. La donnée linguistique mise en avant apporte simplement l'appui indispensable à toute propriété ou trait pour que celle-ci ou celui-ci puisse mériter le statut de sémantique. Elle est, en somme, le garant que la propriété ou le trait en question n'est pas arbitraire, mais que sa pertinence sémantique est établie, non pas par la science (non linguistique), mais bien par le langage.

Nous partirons donc de la question suivante : des noms comme odeur, parfum, senteur, etc., sont-ils abstraits ou concrets? La question n'est pas inutile, dans la mesure où y répondre peut susciter embarras et hésitation. Parler des odeurs comme d'entités abstraites peut sembler insatisfaisant, de même que l'on peut être réticent à les qualifier de concrètes. On notera ici que des informateurs (David et al. 1997) parlent des odeurs comme d'entités abstraites et

3. Exception notable, l'article de Boisson (1997), qui étudie la dénomination des odeurs dans un échantillon de 60 langues et de 9 familles de langues, et qui livre ainsi de nombreuses informations véritablement linguistiques sur les odeurs.

4. Pour celle des verbes, voir l'étude de Theissen (ce volume) sur sentir.

5. Pour une application aux noms d'événements, voir Van de Velde (2006) ; pour les noms de couleurs et pour le mouvement, voir Kleiber (2010 \& à par. a). 
qu'ils éprouveraient sans doute plus de mal à les caractériser comme des entités concrètes :

(1) C'est un parfum, quelque chose d'abstrait que l'on peut sentir. (op. cit. : 55)

Tout dépend évidemment de ce que l'on entend par abstrait, les définitions de l'opposition abstrait/concret donnant lieu, comme l'on sait, à des critères divergents, pouvant conduire au paradoxe de noms à la fois abstraits et concrets (Kleiber, 1994a : chap. 3). Mais, cela a toutefois un avantage, celui de faire émerger, lorsque l'on applique les différents critères à un même type de noms, des traits sémantiques généraux de ce type. Ainsi, notre enquête ne va pas nous amener seulement à répondre à la question du caractère abstrait ou concret des termes d'odeurs, mais elle nous conduira également à aborder d'autres questions essentielles pour une sémantique des odeurs, comme celle, décisive selon nous, du statut de noms comme odeur, parfum, senteur, etc. : s'agit-il ou non de noms de propriété ?

Notre parcours comportera trois parties d'inégales grandeur et importance correspondant aux trois oppositions les plus généralement relevées pour définir la distinction noms abstraits/noms concrets :

- matériel/immatériel;

- accessible aux sens/inaccessible aux sens ;

- autonomie référentielle/non autonomie référentielle.

Nous traiterons très rapidement des deux premières oppositions dans la première partie de notre travail. Nous aborderons plus longuement la troisième plus difficile et beaucoup plus complexe que les deux autres - dans la deuxième partie. L'étude de cette troisième opposition nous conduira, directement, à répondre à la question cruciale du statut du nom odeur : s'agit-il, comme il est dit habituellement, d'un nom de propriété ?

\section{ODEURS : IMMATÉRIELLES, MAIS ACCESSIBLES AUX SENS}

\subsection{Matériel/immatériel}

À un premier niveau, on considère comme concret un nom qui renvoie à une entité matérielle et abstrait celui dont le référent est immatériel. Seront ainsi réputés concrets des noms comme homme, sapin, sable, table, etc., et abstraits des substantifs comme jalousie, explosion, idée, etc. On admet généralement que ce qui caractérise une entité matérielle, $c^{\prime}$ est qu'elle occupe une certaine portion d'espace et possède une forme - intrinsèque pour les substantifs comptables (sapin, homme), contingente pour les substantifs massifs $(\text { sable, vin })^{6}$ - ce qui

6. Nous laissons de côté ici la caractéristique temporelle : les occurrences matérielles sont conçues comme pouvant être les mêmes à deux moments différents. 
implique diverses propriétés perceptuelles et interactionnelles (taille, densité, poids, couleur, on peut les voir, les toucher, les manipuler, etc.).

Si l'on considère les odeurs sous cet angle, il est clair que ce sont plutôt des entités abstraites, ce que reflète la formulation (1) supra. Cela explique également que le nom odeur soit incompatible avec des noms et des prédicats qui impliquent la matérialité :

(2) *la matière d'une odeur / *la forme d'une odeur / *les dimensions d'une odeur

(3) *Quelle est la substance de cette odeur ?

(4) *une odeur en poudre/en grains/en liquide/en gaz

ou avec des adjectifs impliquant la matière ou la forme, etc. :

(5) ?une odeur rouge/grande/ronde/large/plane (dans le sens «matériel »), etc.

(6) *une odeur gazeuse

De même, on ne peut pas :

(7) *palper, peser, mesurer, toucher, soulever, etc. une odeur.

Certains emplois semblent cependant contredire ces observations. En effet, la combinaison du nom odeur avec les quantificateurs spécifiques de la massivité est certes rare, mais cependant possible, sous certaines conditions. C'est le cas dans l'exemple (8) :

(8) Le premier hochet de bébé est souvent le foulard. Facile à manipuler, coloré et léger, on peut y ajouter un peu d'odeur (parfum de maman, huile essentielle, essence de vanille, etc.). (http:// professionassmat.free.fr/l'odorat.htm)

Ce dont il s'agit ici, c'est une substance odorante (parfum, huile essentielle, etc.) que l'on peut quantifier : on peut en ajouter, par exemple, quelques gouttes ou, si son conditionnement le permet, quelques pulvérisations. Il arrive que la nature en produise :

(9) Dès le lendemain de notre arrivée, le jour de Pâques, après le sermon s'il faisait beau temps, je courais jusque-là, voir [...] la rivière qui se promenait déjà en bleu ciel [...] cependant que çà et là une violette au bec bleu laissait fléchir sa tige sous le poids de la goutte d'odeur qu'elle tenait dans son cornet. (Proust, Du côté de chez Swann, 1954)

Ce qui est curieux, c'est que cette dualité sémantique, bien connue pour le nom parfum, soit passée largement inaperçue pour le nom odeur, à telle enseigne que le TLF et le Petit Robert ne l'évoquent même pas. Pour expliquer cette curieuse omission, on peut avancer deux raisons. 
D'abord une raison contingente : les substances odorantes, que l'on peut manipuler et dont on peut faire varier la quantité en suspension dans l'air, sont presque toujours des artefacts qui, tous, produisent une sensation agréable sur notre odorat (à quoi bon en fabriquer de désagréables ?) et c'est le mot parfum que l'on emploie pour les désigner. Le mot odeur est donc rarement utilisé pour désigner une substance matérielle. Mais, il y a une autre raison qui explique l'occultation de cet emploi. Imaginons, en effet, une maman qui, soucieuse de développer le sens olfactif de son bébé, suit le conseil donné dans (8). Après avoir imbibé le foulard de quelques gouttes de parfum, elle pourra évaluer le résultat de l'opération en disant :

(10) J'ai mis trop d'odeur. / Je n'ai pas mis assez d'odeur.

Or, son odorat ne peut lui fournir aucune indication quantitative. Ce qu'elle perçoit, c'est en fait l'intensité de l'odeur. Mais, à partir de l'intensité perçue, elle procède à une inférence concernant la quantité de substance odorante qu'elle a versée, tant il semble naturel de penser que l'intensité perçue varie en proportion de la quantité de substance odorante présente dans l'air ambiant ${ }^{7}$.

En tout état de cause, cet emploi du nom odeur pour désigner une substance matérielle est très occasionnel et, contrairement à ce que l'on constate pour le nom parfum, ne s'est pas imposé de façon stable.

Mis à part ceux qui font intervenir des quantificateurs de massivité, il y a d'autres emplois qui jettent un doute sur l'immatérialité du référent du nom odeur. Celui-ci est, en effet, souvent combiné avec des adjectifs qui appartiennent au registre de la matière ${ }^{8}$, comme le montrent les exemples suivants :

Sur la frise en bas des forêts

Le brouillard échappé des larmes

L'odeur rugueuse des cigares

(Reverdy, Main d'auvre, (1913-1949)/1949)

Nous causions sur les chemins poussiéreux où rôdait l'odeur râpeuse des figuiers. (de Beauvoir, Mémoires d’une jeune fille rangée, 1958)

Le phénomène de synesthésie, dimension inhérente à toute perception (Candau, $2000: 30$ ), permet de rendre compte de ces emplois et, dans beaucoup de cas, une telle réponse peut suffire, surtout si on tient compte de la pauvreté - maintes fois soulignée dans la littérature consacrée aux odeurs - du lexique olfactif, qui nécessite le recours au vocabulaire $\mathrm{d}^{\prime}$ autres domaines sensoriels lorsqu'il s'agit d'exprimer une expérience olfactive particulière. Il n'est cependant pas inutile, nous semble-t-il, d'examiner de plus près quelques-unes de ces combinaisons,

7. C'est d'ailleurs sur cette intuition que l'on se fonde pour détecter la source d'une odeur : plus ça « sent fort », plus on se rapproche de la source.

8. Sur la question des adjectifs combinés avec odeur, voir Vassiliadou \& Lammert (ce volume). 
notamment celles, fréquentes, où les odeurs sont qualifiées d'âcres, râpeuses, rugueuses, brûlantes, chaudes, etc. Deux explications peuvent être envisagées.

Dans certains cas, c'est le vecteur des odeurs qui possède la propriété dénotée par l'adjectif : ainsi, une odeur ne saurait être qualifiée de chaude ou de brûlante que si l'air qui la véhicule est lui-même chaud ou brûlant. Et, si on attribue ces propriétés aux odeurs, c'est parce qu'il est impossible de les distinguer de l'air qui les transporte. L'exemple (13) fournit une bonne illustration de ce phénomène :

Tantôt des bouffées de neige blanchissaient la pelouse, et tantôt des irrigations abondantes effaçaient ce tapis et le dissipaient en une fumée dont l'odeur froide allait à travers les fentes des portes glisser le frisson. Triste soirée d'hiver. (Gozlan, Le notaire de Chantilly, 1836)

C'est dans cette perspective qu'il faudrait entreprendre, pour les autres sens notamment pour le goût qui, comme le rappelle J. Candau (2000: 35), partage avec l'odorat ce que D. Ackermann (1990 : 176) appelle un même puits d'aérage l'analyse des adjectifs non olfactifs combinés avec les odeurs. Cela conduirait sans doute à relativiser le rôle des métaphores, systématiquement invoquées pour expliquer « rhétoriquement » les synesthésies.

Il faut aussi tenir compte des cas, également fréquents, où, par métonymie, on attribue à une odeur une propriété de sa source. C'est le cas dans l'exemple (14) :

Parfois au milieu des terreaux et des sèves, sur la surface bien fondue de leur parfum, on percevait l'odeur granuleuse, artisane et humble de la sciure de bois. (Malegue, Augustin ou le maitre est là, 1933)

Ni l'air ni l'odeur qu'il transporte ne peuvent être à proprement parler granuleux, puisque cette caractéristique est propre aux entités visibles et palpables. Ici, on attribue à l'odeur une propriété de la substance dont elle émane, la sciure de bois ${ }^{9}$. La motivation métonymique est d'ailleurs confirmée par les deux autres adjectifs (artisane, humble) associés au nom odeur.

La combinaison de modificateurs matériels avec le nom odeur n'infirme donc pas l'idée que les entités visées par ce mot sont conçues comme immatérielles. Cette conclusion peut sembler triviale ; elle n'est cependant pas sans importance, car elle nous met en garde contre le risque de confusion entre le sens que revêt le mot odeur dans le langage ordinaire et celui que lui attribue le langage scientifique, qui envisage les odeurs sous l'angle de leur réalité physique, c'està-dire comme composées de molécules, au même titre que n'importe quel corps. On peut donc légitimement conclure, nous semble-t-il, que, pour ce qui est de la

9. Granuleux = Qui n'est pas lisse d'aspect ; dont la surface présente des irrégularités arrondies. (TLF) 
dimension abstractive matériel/immatériel, les odeurs, parce que n'ayant pas de matière, se placent clairement du côté de l'immatériel, donc de l'abstrait.

\subsection{Accessible aux sens vs. inaccessible aux sens}

La deuxième conception de l'opposition abstrait/concret s'articule sur l'accessibilité aux sens : sont concrètes les entités accessibles aux sens, sont abstraites celles qui ne le sont pas. Elle ne coïncide pas avec la conception précédente, puisqu'il existe des référents immatériels accessibles aux sens : nous pouvons voir les couleurs, les formes, vérifier par le toucher la dureté de telle ou telle matière, entendre tel ou tel bruit et, bien évidemment, sentir telle ou telle odeur. Envisagées sous cet angle, les odeurs se placent donc clairement du côté des entités concrètes. Elles n'existent - est-il besoin de le rappeler ? - que parce qu'elles peuvent être perçues par nos organes olfactifs.

\section{AUTONOMIE VS. NON AUTONOMIE}

La troisième dimension ontologique où se définit l'opposition abstrait/concret met en avant l'autonomie ou la non-autonomie référentielle de l'entité. Elle n'est pas sans rapport avec la première (la dimension matériel/immatériel), ainsi qu'on le verra, et nous conduira, comme annoncé, à nous interroger sur la caractérisation des noms d'odeur comme noms de propriété.

\subsection{Ontologie classique}

On partira de l'ontologie classique et de la différence qu'elle établit entre les propriétés et les objets porteurs de ces propriétés. R. Descartes parle d'objets extérieurs possédant des qualités auxquelles chacun de nos sens donne accès :

J'avois expliqué assez particulièrement toutes ces choses dans le traité que j'avois eu ci-devant dessein de publier. Et ensuite j'y avois montré [...] comment la lumière, les sons, les odeurs, les goûts, la chaleur, et toutes les autres qualités des objets extérieurs y peuvent imprimer diverses idées, par l'entremise des sens. (Descartes, Discours de la méthode, 1637 : 184-185)

Cette opposition interne/externe se laisse traduire en termes d'autonomie : les " objets extérieurs » existent pour eux-mêmes, mais non leurs propriétés qui, elles, ne sont pas ontologiquement autonomes :

Tout ce qui est, c'est-à-dire qui subsiste dans la réalité effective [...] relève de l'un ou l'autre des genres suivants : soit cela est et subsiste sur quelque chose d'autre, comme propriété de cette chose, soit ce n'est pas une simple propriété sur quelque chose d'autre, mais cela subsiste, comme on a coutume de le dire, pour soi. La couleur, l'odeur, le poids d'un corps nous fournissent des exemples du premier genre ; car toutes ces choses sont quelque chose de réel-effectif, qui ne subsiste pourtant pas pour soi. Mais seulement sur quelque chose d'autre, à savoir en l'occurrence le corps, et alors assurément comme propriété de celui-ci. [...] Les réalités du premier genre, les 
philosophes ont coutume de les appeler d'un mot latin adhérences, celles du dernier genre substances. (Bolzano, cité par Benoist, 2003 : 141)

Cette impossibilité de subsister par soi-même, n'est pas seulement l'apanage des propriétés et, lorsque P. Strawson (1977) divise les concepts en deux catégories suivant qu'ils sont autonomes ou non, il range aussi les événements (explosion, course, mort, etc.) parmi les non autonomes. Les concepts autonomes, auxquels répondent les substantifs catégorématiques, ont des occurrences qui ne nécessitent pas l'existence d'autres occurrences alors que les occurrences des non autonomes, qui, à la forme nominale, correspondent aux substantifs syncatégorématiques (Kleiber 1981), supposent l'existence d'une occurrence d'un autre concept. Une occurrence de chien ne suppose pas l'existence d'une occurrence d'un autre concept alors qu'une occurrence de blancheur ou d'explosion n'existe que s'il y a une occurrence d'un autre concept, c'est-à-dire que s'il y a quelque chose qui est blanc et quelque chose qui explose.

\subsection{Aliénation possible ou non}

On pourrait être tenté, à ce niveau, d'associer l'autonomie ontologique au concret et la non autonomie ontologique à l'abstrait, en se fondant sur l'impossibilité d'avoir dans la réalité des événements sans arguments et des propriétés sans support. Événements et propriétés seraient donc « abstraits » dans le sens où, ne subsistant pas par eux-mêmes, il faut les « abstraire », c'est-à-dire les dissocier de ce qui rend possible leur manifestation.

On peut s'en tenir là mais, ce faisant, on se prive de voir ce qui rend impossible ladite abstraction ou dissociation et on méconnaît le fait qu'il y a des entités ontologiquement non autonomes que l'on ne qualifierait pas pour autant d'entités abstraites: celles qui constituent des parties d'un tout et qui n'existent donc que dans la mesure où le tout dont elles sont une partie existe aussi. On aura reconnu les entités correspondant aux noms méronymiques comme tête, guidon, etc., dont une occurrence particulière nécessite bien l'existence de l'occurrence particulière d'une autre entité (un corps, un vélo, etc.), mais que l'on ne rangera pas pour autant avec les entités abstraites ${ }^{10}$.

La raison est donnée par E. Husserl et la distinction qu'il fait entre parties dépendantes et parties indépendantes : ce qui fait la différence entre une propriété et une " partie » comme la tête d'un homme est que la seconde, mais non la première, peut être détachée, aliénée par rapport à l'entité dont elle dépend :

On peut assurément se représenter une tête séparée de l'homme auquel elle appartient, on ne peut se représenter de cette manière une couleur, une forme, etc., elles ont besoin

10. Mel'čuk \& Polguère (2008) placent les noms de < partie de > dans la classe plus large de ce qu'ils appellent quasi-prédicats, qui rassemble aussi bien des noms comme mari, idiot, gaucher, ministre, autocar, marteau, etc. Comme ils le soulignent dans leur abstract (op. cit. : 99), «l'ensemble des quasi-prédicats d'une langue est très hétérogène et chaque type de quasi-prédicat pose ses propres problèmes au niveau de la modélisation ». 
d'un substrat, dans lequel on les remarque sans doute exclusivement, mais dont elles ne pourront être séparées. (Husserl, 1962 : 24)

Cette impossibilité d'aliénation fournit un nouveau critère définitoire des noms abstraits : seront ainsi considérés comme abstraits ceux qui renvoient à des entités qui ne sont pas détachables ou dissociables de l'entité dont elles dépendent. Leur abstraction réside dans le fait que leur prise en compte ne peut se faire de façon isolée, dissociée. Inversement, seront réputés concrets non seulement tous les noms renvoyant aux entités référentiellement autonomes mais aussi tous les noms de " parties » (ou méronymes) - comme tête, guidon, etc. - que l'on peut concevoir séparées du tout dont elles font partie. Les noms de propriété comme beauté, tristesse, etc. et les noms d'événement, comme explosion, danser, etc., en revanche, seront classés comme abstraits ${ }^{11}$.

Dans ce cadre, toutes les entités concrètes de la première conception (matériel/immatériel) restent des entités concrètes, mais l'inverse n'est pas nécessairement vrai : il n'est pas acquis que toutes les entités immatérielles, donc abstraites dans le premier sens, deviennent des entités abstraites dans le second sens. Rien ne dit notamment que les odeurs, immatérielles, sont des entités du type de beauté ou du type d'explosion.

Pour le moment, il nous faut expliquer dans quelles conditions le détachement est possible. Si on peut détacher en imagination la tête d'un homme ou en dessiner une image, $c^{\prime}$ est précisément parce qu'elle comporte les traits de forme et de matière requis pour une telle aliénation. La représentation, soit réelle, soit seulement mentale, repose sur la perception visuelle et, à ce titre, engage les traits ontologiques de forme. Avec beauté ou danse, une telle représentation exige que l'on conserve les entités-supports, parce que ce qui fait la spécificité d'une occurrence de beauté ou d'une occurrence de danse engage fondamentalement les « formes » de ces entités.

\subsection{Détour par les couleurs et les formes}

Avant de voir ce qu'il en est des odeurs, nous ferons un petit détour par les couleurs et par les formes.

Il ressort nettement de la citation de B. Bolzano que les couleurs sont conçues comme des propriétés (des adhérences) qui ne subsistent que sur quelque chose d'autre et E. Husserl, pour la même raison, en fait des contenus dépendants. La cause paraît donc entendue. Quelques petites précisions ne sont toutefois pas inutiles.

Certes, on ne peut, si une voiture est bleue, par exemple, enlever (littéralement) la couleur sans enlever une partie concrète du support, la peinture qui est

11. C'est la position, par exemple, de Flaux \& Van de Velde (2000) qui parlent de noms abstraits intensifs pour les noms de propriétés et de noms abstraits extensifs pour les noms d'événements. 
bien une substance. Par contre, on peut fort bien (se) représenter la couleur de la voiture, donc le bleu, sans forcément (se) représenter la voiture. La preuve ? Les nuanciers des catalogues. Certes, il reste un support, la surface du papier, qui elle-même a besoin d'un support tridimensionnel (la feuille de papier) mais, cette fois-ci, la couleur en question n'est appréhendée, ni comme une propriété de la voiture, ni comme une propriété de la feuille de papier qui lui sert de support. Elle est donc bel et bien abordée en tant qu'elle-même, c'est-à-dire en tant que couleur ${ }^{12}$. Une couleur n'apparaît donc pas toujours comme la propriété d'un objet (Kleiber 2007, 2010, à par. b).

Prenons encore le cas d'un triangle (géométrique). Il ne peut exister tel quel dans la réalité, puisque, déjà au niveau de sa forme, il faut faire abstraction de l'épaisseur (c'est-à-dire de la surface) des lignes ou traits qui marquent ses trois côtés. Et sa bidimensionnalité a besoin, comme toutes les surfaces, d'un support tridimensionnel matériel. Il résulte donc nécessairement d'un processus d'abstraction. Cependant, il n'est abstrait que parce qu'il n'a pas de matière. Mais, si je puis me le représenter, $c^{\prime}$ est parce qu'il a des formes propres. Il ne se trouve pas engagé par rapport à une autre occurrence que l'on serait obligé de faire apparaître si on (se) le représente par une image ou mentalement. Alors que, si l'on prend des entités comme beauté et course, il est nécessaire, pour se les représenter, de se représenter aussi des entités qui sont belles ou qui courent. Elles ont donc un statut ontologique différent de celui des couleurs et des formes.

Cette mise au point nous semble très importante, parce qu'elle permet d'éviter les équivoques fréquentes à ce niveau sur ce que l'on entend par occurrences référentiellement autonomes et occurrences référentiellement non autonomes.

\subsection{Application aux odeurs : odeur est-il un nom de propriété ?}

On peut maintenant voir ce qu'il en est - sur ce point et, bien entendu, sur ce point seulement - des odeurs. De prime abord, comme il ressort de l'ontologie classique (cf. Bolzano supra), les odeurs semblent bien dépendantes d'une autre entité dont on ne peut les détacher, leur source. Et, comme manifestement, elles ont un statut très différent des événements, il semble logique de les ranger parmi les propriétés (David, 2002 : 95 ; Lenclud, 2006 : 10). Certes, on ne peut leur appliquer le test de la représentation concrète (par une image) ou mentale autonome, puisqu'elles ne présentent pas de traits perceptibles visuels, donc susceptibles de représentations par des formes, mais ceci n'implique pas, pour autant, qu'elles soient référentiellement non autonomes.

12. La propriété dispositionnelle être rouge est dûment décollable, à la façon d'un timbre que l'on décolle de l'enveloppe [...]. C'est cette opération d'abstraction qui permet à la société humaine de mettre en rapport des objets n'entretenant entre eux aucune relation autre que le partage d'une même couleur. (Lenclud, 2006 : 10) 


\subsubsection{Arguments en faveur de la dépendance ontologique des odeurs}

Les arguments plaidant apparemment pour leur non autonomie ontologique et donc leur statut de propriété sont de plusieurs ordres.

Il y a tout d'abord l'argument ontologique. Une odeur semble ne pouvoir exister sans sa source. G. Lenclud qui, comme nous l'avons vu supra, conclut au " décollement " possible des couleurs, refuse en revanche une telle séparation pour les odeurs :

[...] Contrairement aux couleurs, les odeurs ne se laissent pas, semble-t-il, détacher par l'esprit des objets supposés par nous être intrinsèquement odorants. (op. cit. : 10)

À cette donnée ontologique fait écho l'argument syntaxique que constitue le $\mathrm{SN}$ l'odeur de $X$, où $\mathrm{X}$ représente la source. Cette construction, relevée par tous ceux qui se sont intéressés aux odeurs, marque iconiquement la dépendance ontologique des odeurs par rapport à $X$ et leur statut de propriété de X (cf. David, $2002: 88)$ :

(15) J'aime l'odeur de cette rose.

(16) J'aime la couleur de cette chemise.

Cet argument syntaxique a une contrepartie pragmatique référentielle : si on enlève le SP de $X$, où $X$ dénote la source, on aboutit (le plus souvent) à une incomplétude qui entraîne des questions du genre $c^{\prime}$ est une odeur de quoi ? ${ }^{13}$, question que l'on peut interpréter comme un indice de la non autonomie référentielle des odeurs.

Il y a ensuite l'argument sémantique déjà évoqué supra : c'est que, dans son emploi de très loin le plus fréquent (celui dont nous nous occupons ici), le nom odeur dénote des entités dont on ne perçoit que l'intensité. Or, à certains égards, le nom odeur se comporte comme les noms de qualité (courage, énergie, audace, etc.), qui sont des substantifs syncatégorématiques typiques. Ainsi, il réagit comme ces derniers au contact de peu de/beaucoup de. Dans les deux cas, c'est une interprétation intensive qui s'impose ${ }^{14}$ :

(17) Cette fleur (a beaucoup/a peu/n'a pas beaucoup) d'odeur. = Cette fleur (sent fort/ne sent pas fort) / (a une odeur forte/a une odeur faible).

(18) Pierre a beaucoup de courage/d'énergie/de patience $/ d^{\prime}$ audace. $=$ Pierre est très courageux/énergique/patient/audacieux.

De fait, un dialogue comme :

$$
\text { ??Combien cette fleur a-t-elle d'odeur ? - Beaucoup. }
$$

13. Dont le correspondant verbal est Ça sent quoi?

14. Cf. Buvet (2001). 
est tout aussi incongru que :

$$
\text { ??Combien Pierre a-t-il de courage ? - Beaucoup. }
$$

En outre, odeur, peut, comme les noms de qualité, apparaître dans la construction un(e) de ces $N$, ce qui est exclu pour les $\mathrm{N}$ massifs :

(21) Il a un de ces courage(s) / une de ces (patience(s)/énergie(s)/audace(s)/...).

(22) Il régnait dans la pièce une de ces odeur(s) !

(23) *Il a un de ces (fric(s)/argent(s)/...)!

(24) *Il y avait une de ces eau(x)!

Du point de vue morphologique enfin, l'existence de l'adjectif odorant (voir aussi pour les odeurs agréables, odoriférant) et de l'antonyme inodore ${ }^{15}$, qui, par leur statut catégoriel (puisqu'il ne s'agit pas dans plantes odorantes d'un pseudoadjectif ou adjectif relationnel, même s'il fonctionne comme adjectif classifiant) milite pour que l'on reconnaisse dans les odeurs des attributs ou propriétés de l'entité dénotée par le nom auquel se rapportent ces adjectifs ${ }^{16}$.

\subsubsection{Contre-arguments}

Ces différents arguments ne sont toutefois pas totalement convaincants.

L'argument ontologique ne s'avère pas pertinent pour faire d'odeur un $\mathrm{N}$ de propriété. Le lien qui lie une odeur à sa source n'est pas du même ordre que celui qui associe une propriété à son support : l'odeur signale indexicalement donc par une relation causale - qu'il y a une substance qui dégage une odeur, ou qui «sent ».

Prenons comme exemple la fumée : elle signale qu'il y a du feu. Autrement dit, c'est un phénomène qui, sur un plan sémiotique, est un indice du feu alors que, sémantiquement, le nom qui la dénote, le substantif fumée, n'est pas un signe indexical, mais bien un signe symbolique ${ }^{17}$. De même, une odeur, en tant que phénomène, est un signe indexical de l'existence de quelque chose qui sent, mais le mot odeur lui-même n'est pas un signe indexical.

Ce qui est intéressant ici, c'est que la relation indexicale qui associe une odeur à une source autorise précisément le détachement refusé aux propriétés ou aux événements. En règle générale d'ailleurs, la notion sémiotique d'index, appelée de façon peut-être plus transparente par certains, symptôme, s'applique

15. Soulignons - c'est révélateur - que l'on n'a pas au positif un odore dont inodore serait l'antonyme.

16. Cet argument morphologique est couramment employé pour les couleurs.

17. De même, les onomatopées renvoient à une entité (le bruit) en relation indexicale avec ce qui est à l'origine du bruit, mais elles ne sont pas elles-mêmes des signes indexicaux, mais des signes iconiques (Kleiber 2006). 
précisément aux cas où l'objet qui est signifié indexicalement n'est pas immédiatement perceptible ${ }^{18}$ ou accessible, comme l'illustre clairement le phénomène de la fièvre qui est le symptôme d'un état anormal. La relation indexicale entre source et odeur, non seulement n'empêche pas d'appréhender l'odeur de façon autonome, mais s'avère surtout pertinente, sémiotiquement parlant, dans les cas d'appréhension détachée. L'argument se retourne donc en faveur de l'autonomie ontologique des odeurs.

L'argument que nous avons rapidement appelé syntaxique, à savoir la construction binominale en $d e$, n'est pas pleinement décisif non plus, pour au moins deux raisons. La première est qu'une telle construction n'est syntaxiquement pas obligatoire pour l'expression d'une occurrence spécifique d'odeur alors qu'elle est exigée pour l'occurrence d'une propriété spécifique. Comme le montre l'énoncé (25) :

(25) Une odeur nauséabonde régnait dans la pièce.

nous pouvons fort bien introduire une occurrence spécifique d'odeur sur le mode indéfini existentiel ou faible, sans mention aucune de la source. Semblable présentation indéfinie d'une occurrence spécifique de propriété n'est pas envisageable. Plus précisément, l'interprétation d'un énoncé comme (26) :

(26) Une grande tristesse régnait au fond des mers.

oblige à chercher dans le contexte (discursif ou situationnel) une entité que l'on puisse concevoir comme le siège du sentiment dénoté par une grande tristesse, donc des êtres capables d'éprouver des sentiments et vivant au fond des mers ou, à défaut, des témoins qui projetteraient sur le fond des mers le sentiment qu'ils éprouvent en le contemplant.

En deuxième lieu, la source d'une odeur particulière peut être différente de l'entité indiquée par le nom du syntagme en de:

(27) Ce citron avait (une odeur de pomme/l'odeur de la pomme/l'odeur d'une pomme/l'odeur des pommes). ${ }^{19}$

(27) montre que l'odeur particulière ressentie est appréhendée indépendamment de la source particulière (ici le citron dont il est question), même si le $\mathrm{N}$ pomme fonctionne comme source, mais au niveau du type et non de l'occurrence particulière.

18. Cf. les interjections et l'exclamation en général qui sont le signe indexical d'une émotion que l'on ne perçoit pas directement (Kleiber 2006).

19. Nous reconnaissons fort volontiers que la première formulation peut paraitre plus naturelle que les trois autres, la deuxième pouvant même poser un problème d'acceptabilité. 
L'argument référentiel paraît plus solide, dans la mesure où il semble bien qu'un SN «nu » avec odeur soit senti comme incomplet et appelle une saturation du type source en réponse à une interrogation du type une odeur de quoi ? :

(28) ?Une odeur régnait dans la pièce. ${ }^{20}$

(29) Une odeur de citron régnait dans la pièce.

Mais en fait cette incomplétude est due à la hauteur abstractive du mot odeur il s'agit d'un terme super-ordonné - et non pas à une incomplétude syncatégorématique, comme le prouve l'ajout d'un modificateur comme nauséabonde, qui, sans rien révéler sur la source, suffit cependant pour garantir à odeur un seuil d'informativité suffisant :

(30) Une odeur nauséabonde régnait dans la pièce.

Les termes super-ordonnés servent à rassembler des catégories basiques et non pas à les identifier (Wierzbicka 1985 ; Kleiber 1994b). On peut fort bien avoir un énoncé tel que :

Une odeur dont je ne pouvais deviner l'origine régnait dans la pièce.

qui prouve explicitement que la non identification de la source ne porte pas atteinte à un emploi spécifique du SN une odeur.

Mais surtout, semblable insuffisance informative guette les emplois référentiels des termes super-ordonnés en général, donc pas seulement du $\mathrm{N}$ odeur. Si l'on prend animal dans un énoncé tel que (32) :

(32) Un animal gambadait dans la cour.

on observe qu'il suscite également une frustration informative qui peut amener une question similaire à l'une de celles suscitées par l'emploi référentiel «nu » $\mathrm{d}^{\prime}$ odeur ${ }^{21}$ :

\section{C'était quoi comme animal ?}

Quant à l'argument sémantique, il n'est pas plus convaincant, car, si les entités dénotées par le $\mathrm{N}$ odeur dans (peu/beaucoup) d'odeur ne sont effectivement pas appréhendées sous l'angle de la quantité, mais bien sous celui de l'intensité, on ne saurait pour autant en faire des $\mathrm{N}$ de propriétés, de même que l'on ne fait pas des sons, par exemple, des propriétés alors qu'ils présentent également la

20. On a bien, comme nous l'a fait remarquer un de nos relecteurs, un $\mathrm{SN}$ « nu » avec odeur dans les tours du type un de ces $N$ (voir supra) : Il régnait dans la pièce une de ces odeurs !. L'exclamation sert ici d'adjuvant qualitatif qui permet à odeur de fonctionner comme s'il y avait un modificateur (voir l'énoncé 30).

21. Mais, soulignons-le, beaucoup moins embarrassante, parce que, en l'occurrence, les termes subordonnés ne manquent pas. 
dimension 'intensité'. La preuve en est que, contrairement aux $\mathrm{N}$ de propriété, le $\mathrm{N}$ odeur se combine difficilement avec un que exclamatif à interprétation intensive $^{22}$ :

$$
\begin{aligned}
& \text { Que de courage/patience/énergie (il lui a fallu)! } \\
& \text { *Que d'odeur! }
\end{aligned}
$$

et ne donne pas lieu non plus, comme eux, à une quantification métaphorique :

$$
\begin{aligned}
& \text { Des tonnes (de courage/d'énergie) } \\
& { }^{*} \text { Des tonnes d'odeur }
\end{aligned}
$$

Son aptitude à figurer dans la construction $u n(e)$ de ces $N$ ne prouve rien, étant donné que, dans cet environnement, on trouve aussi des $\mathrm{N}$ qui désignent des entités autonomes :

(38) Il a une de ces bagnoles/baraques/costard(s)/...

Bref, odeur se distingue clairement des noms de qualité.

L'argument morphologique appelle plusieurs précisions. En premier lieu, si l'on reprend l'exemple des couleurs, il ne saurait être déterminant. En effet, l'existence d'adjectifs de couleurs ne fait pas automatiquement des couleurs, et donc des noms qui les dénotent (comme le blanc, le bleu, le bleu-clair, etc.), des propriétés (Kleiber 2007, 2010). Mais, même si l'on fait abstraction de ce contreargument, on peut faire valoir qu'il n'y a pas, comme du côté des couleurs, des adjectifs d'odeurs. Certes, il y a odorant, mais ce terme, à cause de son côté " actif ", que manifeste son opposition déséquilibrée à inodore, ne signifie pas exactement 'qui a une odeur'. Par ailleurs, si les odeurs étaient des propriétés, le rapport d'odorant à odeur devrait être le même que celui qui existe entre les adjectifs de couleur et les noms de couleur. Or, ce n'est pas le cas : odorant semble formé à partir du $\mathrm{N}$ odeur alors que, dans le domaine des couleurs, ce sont les adjectifs (par exemple bleu) qui donnent naissance par dérivation impropre aux $\mathrm{N}$ de couleur (cf. le bleu), et non le contraire. La morphologie n'est donc pas un auxiliaire fiable pour la thèse des odeurs conçues comme des propriétés, ontologiquement dépendantes de la substance qui en est la source.

Pour le moment, nous n'avons fait que réfuter cette thèse. Voici maintenant des arguments qui militent pour l'indépendance des odeurs et leur statut d'entités autonomes.

\subsubsection{Perception et dissociation}

Première observation : on ne perçoit pas de la même façon le rapport des couleurs à leur support que celui des odeurs à leur source. Si une voiture est 
bleue, on perçoit la voiture elle-même en même temps que sa couleur. La couleur de la voiture peut donc être perçue comme une propriété de la voiture. Mais, si on perçoit une odeur de frites, on ne perçoit pas nécessairement les frites, et on n'en a d'ailleurs pas besoin. C'est donc bien l'odeur seule qui est l'objet de la perception. C'est évidemment la différence de sens activé qui est à l'origine de cette différence : je ne peux percevoir avec le «nez » que l'odeur des frites, et non les frites elles-mêmes, alors qu'avec la vue je perçois en même temps la voiture et sa couleur. Ce truisme n'est pas sans conséquence pour nous : il implique, par avance, que les odeurs ne peuvent être perçues que détachées de l'objet-source, puisque celui-ci ne peut être perçu que par un sens autre que l'odorat.

\subsubsection{Manifestations linguistiques de la dissociation}

Notre deuxième argument est linguistique : les emplois du $\mathrm{N}$ odeur montrent clairement que les odeurs sont conçues comme existant en dehors de la substance dont elles émanent, alors que les couleurs sont vues comme existant sur la ou une surface de l'objet porteur même.

On peut le montrer de deux façons. Premièrement, en notant une différence de compatibilité avec les prépositions à et $d e$. On n'a pas, pour les odeurs, un syntagme binominal du type :

Un $\mathrm{N}$ d'odeur + modificateur

semblable à celui dont on dispose pour les couleurs :

(40) Une robe de couleur bleue.

(41) *Un citron d'odeur sucrée.

(42) *Un gâteau d'odeur de citron.

C'est la préposition à qui s'impose :

(43) Un citron à l'odeur sucrée.

(44) Un gâteau à l'odeur de citron.

Selon P. Cadiot (1997: 63), ’̀ « ne traduit rien de plus que l'attribution ("exter$\left.n e^{\prime \prime}\right)$ d'un élément de caractérisation » et " maintient l'extériorité des propriétés spécifiantes construites à partir du domaine de N2 » alors que de « vise l'identification ("interne"), ("essentielle") et incorpore dans la représentation suscitée par N1 le domaine de spécification référentielle que permet de fixer N2 ». Les odeurs sont donc conceptualisées comme des éléments indépendants de leur source et non pas comme des éléments qui font un avec elle, comme les couleurs avec leur objet porteur.

D'autre part, le lieu d'existence des odeurs n'est pas l'objet source, comme l'objet porteur l'est pour les couleurs, mais l'air qui nous environne. Les odeurs flottent, se répandent, montent, s'élèvent... dans l'air. M. Vuillaume (ms) l'a 
« schmilblické » en soulignant que des phrases banales comme (45-48) supposent la dissociation spatiale :

D'où vient cette odeur?

Le vent du soir apportait des odeurs printanières.

Tout baignait dans une odeur de friture.

Une odeur de fleurs d'oranger avait envahi la pièce/s'était répandue dans la pièce.

On peut expliciter plus avant cette observation en notant différents points, souvent entremêlés :

- le lieu d'existence ${ }^{23}$ des odeurs est l'air et, par conséquent, l'espace, à savoir les endroits englobants dans lesquels on se trouve (cf. des odeurs de cuisine flottaient dans l'air) ;

- les odeurs n'y occupent pas de place précise mais imprègnent l'air, l'emplissent (cf. l'odeur d'hier au soir continue d'imprégner l'air), se répandent, se diffusent dans l'air ${ }^{24}$ (cf. l'odeur aromatique du café s'exhale, s'épand) et, du coup, comme l'air lui-même, elles englobent (environnent) le sujet qui les perçoit (cf. Madame Gide y besogne dans une odeur entêtante de pétrole) ;

- leur mode d'existence dans l'air : elles n'y sont pas immobiles (cf. leur côté volatil), mais y flottent (cf. les premières odeurs de l'automne flottaient jusque dans la chambre), s'y déplacent de l'objet-source (cf. les odeurs émanent, proviennent, se dégagent de, s'exhalent de $X$, etc.) jusqu'à celui qui les perçoit (cf. les odeurs de la ville parviennent à nos narines) ou bien sont envisagées comme transportées par l'air (en somme, c'est l'air qui se déplace et qui les apporte avec lui jusqu'à nos organes olfactifs) ${ }^{25}$ (cf. la brise m'apportait une odeur d'herbe fraîche).

Ces différentes manifestations de la dissociation spatiale odeur-source confirment donc qu'un nom comme odeur n'est pas un nom de propriété.

\section{DEUX RÉSULTATS POUR CONCLURE}

Premier résultat : aussi bien pour ce qui est de la conception fonctionnelle que de celle mettant en jeu l'accessibilité aux sens, les odeurs se placent du côté

\footnotetext{
23. Nous voulons dire par là qu'elles se manifestent hors de l'objet-source. Mais, comme nous l'a fait remarquer C. Schwarze, que nous remercions, des énoncés comme Le bouchon sent le moisi ou L'eau de Javel sent mauvais donnent à penser que l'odeur, dans ces cas, est d'une certaine manière localisée dans le bouchon et dans l'eau de Javel.

24. Boisson (1997 : 37) parle à ce propos de la variable du volume, « c'est-à-dire le fait qu' une odeur se diffuse largement dans toute une zone, et ceci indépendamment de son intensité (voir Roudnitzka (1990) à propos des parfums) ». Il illustre ce fait en signalant que le terme hawaïen puîa peut être défini comme "sweet-smelling, diffused, as fragrance; permeated with perfume".

25. Citons ici à nouveau Boisson (op. cit. : 38) qui a relevé que certaines odeurs en hawaïen « sont spécifiées comme apportées par le vent, par un déplacement d'air (ainsi les gloses 'wind-blow fragrance, wafted fragrance' pour mâpu et moani)».
} 
du concret, et non de l'abstrait. Si l'on envisage, par contre, la dimension de la matérialité, elles apparaissent bien entendu du côté de l'abstrait, puisqu'elles n'ont ni formes ni matière. Rien d'étonnant à cela, puisqu'elles n'engagent qu'un seul sens, l'odorat, alors que les objets concrets, si l'on prend les exemples canoniques comme chien, sable, etc., peuvent engager les cinq sens (vue, toucher, odorat, ouie, goût) et apparaissent donc éminemment plus... concrets de ce point de vue-là.

Second résultat, sans doute plus important que le premier: les termes d'odeurs ne sont pas des noms de propriété comme on pourrait le penser et comme il est dit bien souvent. Quel est alors leur statut ? La suite au prochain...

\section{Références}

[Petit Robert] Robert P. (1982), Le petit Robert. Dictionnaire alphabétique \& analogique de la langue française, Paris : Société du Nouveau Littré.

[TLFi] http://atilf.atilf.fr/

ACKermann D. (1990), Le livre des sens, Paris : Grasset.

BenoIST J. (2003), "Propriété et détermination : sémantique et ontologie chez Bernard Bolzano ", Philosophiques 30 (1), 137-148.

Boısson C. (1997), "La dénomination des odeurs : variation et régularités linguistiques ", Intellectica 24, 29-49.

BUVET P.-A. (2001), "Les déterminants intensifs ", in X. Blanco, P.-A. Buvet \& Z. Gavriilidou (éds), Lingvisticæ Investigationes Supplementa $\mathrm{n}^{\circ} 23$ : Détermination et formalisation, Amsterdam/Philadelphia : John Benjamins, 101-114.

CADIOT P. (1997), Les prépositions abstraites en français, Paris : Armand Colin.

CANDAU J. (2000), Mémoires et expériences olfactives. Anthropologie d'un savoir-faire sensoriel, Paris : Presses Universitaires de France.

COTTIER-FABIAN E. (2007), "La description des parfums en français et en anglais contemporains ", in A.-M. Laurian (éd.), Les cinq sens et les sensations, Berne : Peter Lang, 35-60.

DAVID S. (1997), "Représentations sensorielles et marques de la personne : contraste entre olfaction et audition ", in D. Dubois (éd.), Catégorisation et cognition : de la perception au discours, Paris : Kimé, 211-242.

DAVID S. (2000), "Certitudes et incertitudes dans les domaines olfactif, gustatif et auditif ", Cahiers du LCPE (Langages, cognitions, pratiques, ergonomie) 4, 77-108.

DAVID S. (2002), “Linguistic Expressions for Odors in French", in C. Rouby et. al. (eds), Olfaction, Taste, and Cognition, Cambridge: Cambridge University Press, 82-99.

DAVID S. et al. (1997), "L'expression des odeurs en français : analyse lexicale et représentation cognitive ", Intellectica 24, 51-83.

DuBoıs D. (éd.) (1997), Catégorisation et cognition : de la perception au discours, Paris : Kimé.

Duboıs D. (éd.) (2009), Le Sentir et le Dire, Paris : L'Harmattan.

Dubois D. (2000), "Categorization as Acts of Meaning: The Case of Categories in Olfaction and Audition”, Cognitive Science Quaterly 1, 35-68.

Duвoıs D. (2006), "Des catégories d'odorants à la sémantique des odeurs. Une approche cognitive de l'olfaction ", Terrain 47, 89-106. 
DuBoIs D. (2007), "From psychophysics to semiophysics: Categories as acts of meaning. A case study from olfaction and audition, back to colors", in M. Plümacher \& P. Holz (eds), Speaking of Colors and Odors, Aspects, Amsterdam: John Benjamins Publishing Company, 67-184.

Duвols D. (2008), "Sens communs et sens commun : expériences sensibles ; connaissance(s) ou doxa ? ", Langages 170, 41-53.

Dubols D. \& Holley A. (éds) (1997), Intellectica $\mathrm{n}^{\circ} 24$ : Olfaction : du linguistique au neurone, Saint Denis : MSH Paris-Nord.

DuBois D. \& RouBy C. (1997), "Une approche de l'olfaction : du linguistique au neuronal ", Intellectica 24, 9-20.

ENGHELS R. (2007), Les modalités de perception visuelle et auditive, Tübingen : Niemeyer Verlag.

FLAUX N. \& VAN DE VeLdE D. (2000), Les noms en français. Esquisse de classement, Paris : Ophrys.

FRANCKEL J.J. (2001), " De l'interprétation à la glose ", Le Gré des Langues 16, 48-65.

FRANCKEL J.-J. \& LEBAUD D. (1995), "Les échappées du verbe sentir ", in J. Bouscaren, J.-J. Franckel \& S. Robert (éds), Langues et langage : problèmes et raisonnement en linguistique. Mélanges offerts à Antoine Culioli, Paris : Presses Universitaires de France, 261-277.

HusserL E. (1962), Recherches logiques, T. 2, Paris : Presses Universitaires de France.

KLEIBER G. (1981), Problèmes de référence. Descriptions définies et noms propres, Paris : Klincksieck.

Kleiber G. (1994a), Nominales. Essais de sémantique référentielle, Paris : Armand Colin.

KLEIBER G. (1994b), "Lexique et cognition : Y a-t-il des termes de base ? ", Rivista di Linguistica 6 (2), 237-266.

KLEIBER G. (2001), "Indéfinis : lecture existentielle et lecture partitive ", in G. Kleiber, B. Laca \& L. Tasmowski (éds), Typologie des groupes nominaux, Rennes : Presses Universitaires de Rennes, 47-97.

KLEIBER G. (2006), "Sur la sémiotique de l'interjection ", Langages 161, 9-23.

KLEIBER G. (2007), "Adjectifs de couleur et gradation : une énigme... < très> colorée ", Travaux de linguistique 55, 9-44.

KLEIBER G. (2010), "Typologie des noms : le cas des noms de couleurs ", in I. Choi-Jonin, M. Duval \& 0 . Soutet (éds), Typologie et comparatisme. Hommages offerts à Alain Lemaréchal, Leuven : Peeters, 249-263.

KLEIBER G. (à par. a), "Dans le <sens> du mouvement : éléments de sémantique conceptuelle du nom mouvement ", Mélanges pour Peter Koch.

KLEIBER G. (à par. b), "Petite sémantique des couleurs des odeurs ", Actes du Vle Colloque International Linguistique contrastive germano-romane et intraromane (Innsbrück, 3-5 septembre 2008).

LANGacker R. (1991), " Noms et verbes ", Communications 53, 103-153.

LAURIAN A.-M. (éd.) (2007), Les cinq sens et les sensations, Berne : Peter Lang.

LENCLUD G. (2006), " La nature des odeurs (remarques) ", Terrain 47, 5-18.

MeL'ČUK I. \& Polguère A. (2008), "Prédicats et quasi-prédicats sémantiques dans une perspective lexicographique ", Lidil 37, 99-114.

PAGNIER T. (2009), Les nominations des couleurs, des bruits et des odeurs par les élèves d'une classe de CE1, Paris : Thèse de Doctorat de l'Université de Paris 3.

RoudnitzKa E. (1990), Le Parfum, Paris : Presses Universitaires de France (3e éd.).

Strawson P. F. (1977), Étude de logique et de linguistique, Paris : Le Seuil. 
TERRAIN 47 : Odeurs, septembre 2006, Ministère de la culture / Maison des sciences de I'Homme.

TERRAIN 49 : Toucher, septembre 2007, Ministère de la culture / Maison des sciences de I'Homme.

Theissen A. (2011), "Sentir : les constructions prédicatives de l'olfaction ", Langages 181, Paris : Armand Colin (ce volume).

VAN DE VELDE D. (2006), Grammaire des événements, Villeneuve d'Ascq : Presses du Septentrion.

VASSILIADOU H. \& LAMmERT M. (2011), " Odeurs et dimension hédonique à travers le prisme des adjectifs ", Langages 181, Paris : Armand Colin (ce volume).

VENDLER Z. (1967), Linguistics in Philosophy, Ithaca: Cornell University Press.

VUILLAUME M. (ms), "Schmilblick".

WiERZBICKA A. (1985), Lexicography and Conceptual Analysis, Ann Arbor: Karoma. 